\title{
Movimiento estudiantil y transformaciones sociales en Chile: una perspectiva sociológica
}

\author{
Nicolas Fleet ${ }^{1}$ \\ Universidad de Cambridge, Cambridge, Reino Unido. \\ Email: ngf22@cam.ac.uk
}

\begin{abstract}
Resumen: Se propone una lectura sociológica de las recientes movilizaciones estudiantiles en Chile, considerando sus demandas en función de las orientaciones e intereses correspondientes a la base social de nueva clase media. Se discuten cuatro hipótesis: primero, la reivindicación de la educación pública puede expresar una crisis de legitimidad; segundo, tal crisis se corresponde con la ampliación y diferenciación de los grupos constitutivos de la nueva clase media (incluyendo la masificación de estudiantes, profesionales e intelectuales en general) en el contexto de las transformaciones de la estructura social en Chile en los últimos treinta años; tercero, la demanda por educación pública se orienta a ejercer control reflexivo sobre las bases de reproducción estructural y simbólica de este grupo y; cuatro, la crítica a la distribución del poder a través de la educación es una plataforma para el planteamiento de un potencial proyecto alternativo de sociedad.

Palabras clave: Movimiento estudiantil, nueva clase media, intelectuales, profesionales, educación superior
\end{abstract}

\section{Student movement and social transformations in Chile: a sociological approach}

\begin{abstract}
This essay proposes a sociological interpretation of the current student movement in Chile, regarding their demands in relation to the orientations and interests of the new middle class. Four hypotheses are discussed: first, the claim for public education could be expressive of a legitimacy crisis; second, such crisis corresponds to the expansion and differentiation of the new middle class (regarding the massification of students, professionals and intellectuals in general) in the context of the transformation of the social structure in Chile during the last thirty years; third, the demand for public education is aligned with the exercise of reflexive control on the structural and symbolic basis of the reproduction of this group and; fourth, the critique of the institutionalization of distribution of power through education constitutes the platform to sustain, from part of this group, a potential alternative project of society.
\end{abstract}

Key words: student movement, new middle class, intellectuals, professionals, higher education 


\section{Movimento estudantil e transformações sociais no Chile: uma abordagem sociológica}

Resumo: Este ensaio propõe uma interpretação sociológica do movimento estudantil atual no Chile, considerando suas demandas em relação às orientações e interesses da nova classe média. Quatro hipóteses são discutidas: a primeira, a alegação para a educação pública pode ser expressão de uma crise de legitimidade; segundo, tal crise corresponde à expansão e diferenciação da nova classe média (em relação à massificação de estudantes, profissionais e intelectuais em geral) na contexto da transformação da estrutura social no Chile durante os últimos 30 anos; em terceiro lugar, a demanda para a educação pública está alinhado com o exercício do controle reflexivo sobre a base estrutural e simbólica da reprodução desse grupo e; em quarto lugar, a crítica da institucionalização da distribuição do poder através da educação constitui a plataforma para sustentar, de parte deste grupo, um projeto alternativo potencial da sociedade.

Palavras-chave: movimento estudantil, nova classe média, intelectuais, profissionais, ensino superior.

$* * *$

Recibido: 01.09.2011
Aceptado: 23.11.2011

\section{Introducción}

El objetivo de este ensayo es esbozar un marco comprensivo amplio, a partir del análisis de las transformaciones sociales que se han venido desarrollando en los últimos treinta años en el país, para poder así, en este contexto, resignificar las orientaciones del actual movimiento estudiantil. Para ello, desarrollaré cuatro hipótesis generales que permiten replantear la coyuntura de la movilización en el contexto mayor que se propone.

Primero, el momento actual expresa una crisis de legitimidad; segundo, el movimiento estudiantil, particularmente en referencia a la educación superior, se corresponde con la ampliación del segmento de nueva clase media (incluyendo la masificación de la categoría intelectuales en relación al trabajo profesional) en Chile. Tercero, las orientaciones del movimiento apuntan a ejercer control reflexivo, a través de medios administrativos, sobre la organización de la educación y de esta manera sobre las bases de formación y reproducción de la nueva clase media; cuarto, la clase de sectores medios y trabajadores intelectuales potencialmente irrumpen para plantear un proyecto alternativo de sociedad.

Antes de comenzar es necesario señalar que la limitación del presente análisis es que se enfoca principalmente en la educación superior, de modo que las mencionadas hipótesis tienden a circunscribirse exclusivamente a este ámbito. 


\section{Crisis de legitimidad}

El primer esbozo consiste en hipotetizar el momento actual como expresión de una crisis de legitimidad. En esta línea, durante 2011 las encuestas de Adimark y CEP han mostrado evidente desafección con la clase política, y ello ya era un fenómeno relativamente patente a partir de situaciones como la baja participación juvenil en elecciones. Los problemas de identificación con el régimen político y con los gobernantes pueden ser efectos de una crisis de legitimidad que, a su turno, refiere a un proceso de mayor duración y alcance. En los términos de Max Weber, quien acuñó el concepto, una crisis de legitimidad plantea una fractura en el esquema de dominación en su conjunto, producida por un grupo social emergente que modifica la identidad de la sociedad a la vez que presiona por mayor participación en la distribución del poder (y a través del poder, del producto económico) y reconocimiento social, conduciendo a la apertura de este esquema, o sea su democratización, o bien a su cierre, es decir exclusión. Como toda dominación que pretende ser legitima se basa en el principio que el grupo dominante ejerce el poder en beneficio de la colectividad, un aspecto fundamental para entender simbólicamente una crisis de legitimidad es que la disputa a la dominación la lleva a cabo el grupo emergente o dominado en nombre de los mismos valores o fines colectivos que han sido insatisfechos por el grupo dominante, de manera de justamente restarle legitimidad a esta clase dominante y llevar entonces a cabo la promesa incumplida (Fleet, 2009). La crítica al modelo educativo en general se comprende en relación a una crisis de legitimidad en la medida que éste no ha traído, desde el punto de vista de la sociedad en su conjunto, ni igualdad ni integración, sino que más bien ha sido el principal mecanismo para perpetuar la división de la sociedad en posiciones dominantes y dominadas, del mismo modo que los otros mecanismos para la distribución de los recursos el sistema, el trabajo y el consumo, también han conducido -notoriamente a través de la precariedad, explotación y endeudamiento- a defraudar el sentido de solidaridad del grupo que sustenta a todo esquema de dominación legítima.

Además, existe un problema de legitimidad política de origen, como ha sido planteado por Manuel Antonio Garretón en los medios de comunicación: las críticas a las instituciones de distribución del poder en el país, partiendo por la Constitución de 1980 y siguiendo por la educación, entonces se concentran en su origen en dictadura, que básicamente amplió las distancias entre la sociedad y los centros del poder político. Como dijo Norbert Lechner (1983), la dictadura en Chile desmontó el incipiente Estado de Bienestar a través de una represiva desmovilización política. Por lo tanto, la crítica al carácter no democrático de la institucionalidad de la educación en Chile se conecta a la crisis de legitimidad de todo el sistema de representación.

Si el momento actual es expresivo de una crisis de legitimidad, significa que un grupo social irrumpe y plantea la posibilidad concreta de ampliar la distribución del poder en el país, con lo cual los escenarios posi- 
bles son la consecuente democratización social y política del esquema de dominación, o bien la reacción del grupo dominante tendiente a hacer más rígido y excluyente el ejercicio del poder. El mencionado descrédito de la clase política en Chile es sin duda un síntoma de aquello, pero no es que solo en el último tiempo se haya producido la alienación entre sociedad y política; tal divorcio ya se había consumado en la época de los gobiernos de la Concertación. Más bien, se trata de la percepción de que la clase política, en tanto administradora más que representante, no es capaz de responder con un mínimo de eficacia a los problemas planteados por la integración de la sociedad, y al ser en consecuencia relativamente funcional a los intereses de la clase dominante, se convierte en parte del problema y en síntoma de la crisis.

\section{Nueva clase media e intelectuales}

El segundo elemento de este contexto es que las transformaciones recientes en la sociedad chilena han conducido a la ampliación y diferenciación de grupos sociales cuya presencia e importancia relativa sería mayor que el poder al que efectivamente tienen acceso de acuerdo a las instituciones que lo distribuyen y legitiman. Por muchos años, digamos entre la segunda mitad de los noventa hasta comienzos de la década de 2000, el debate sociológico sobre el país tenía un lugar común en la identificación de una crítica difusa, por ejemplo en la idea del malestar, hacia un sistema, el neoliberal, que tiende a la desintegración social. Sin embargo, no había claridad de quiénes o cuáles serían los actores sociales que llevarían a la práctica esta crítica en la forma de un proyecto alternativo de sociedad. En cambio hoy es posible ver en los estudiantes una manifestación concreta de esta crítica y también la incipiente formulación de un proyecto de sociedad, basado principalmente en la aspiración de impulsar democratización social por medio de la educación con carácter público y abolir la institucionalidad heredada de la dictadura para este ámbito. La pregunta es si las recientes movilizaciones estudiantiles son parte, como una especie de vanguardia, de un actor social más amplio.

Por ejemplo, Gabriel Salazar ha sostenido en los medios de comunicación que el movimiento estudiantil es expresión o ejemplo de movimientos ciudadanos, los que en su estado maduro se definen por la práctica de la autogestión, o sea la producción de los medios para reproducir el movimiento y satisfacer sus fines (si es que los tienen, es decir si es que pueden ser descritos de acuerdo a modelos teleológicos de acción colectiva). Los movimientos ciudadanos vienen a ocupar el espacio que una vez tuvieron el movimiento democrático y de derechos humanos, durante la década de los ochenta, y el movimiento obrero, durante gran parte del siglo XX, en la definición del conflicto central de la sociedad chilena. La propuesta de Salazar hace sentido en la medida que los movimientos estudiantiles han actuado con autonomía respecto de los partidos políticos y tampoco pueden ser fácilmente endosados a actores de clase tradicionalmente definidos. Sin embargo, dudo que el futuro desarrollo de este movimiento esté, 
como propone Salazar, en el despliegue de prácticas autónomas para resolver los problemas que plantea, cuando la demanda justamente ha sido recuperar el rol activo del Estado en la definición del contenido y en la provisión de la educación pública y gratuita. Para reivindicar el status de la educación como derecho y no como mercancía el papel de la burocracia estatal es reclamado como indispensable. Es decir, la potencialidad del movimiento estudiantil no se restringe en la práctica ni en el análisis al poder de la acción colectiva propiamente tal, el cual de manera cíclica se despliega públicamente y luego se retrae hacia la sociedad civil, sino que en su capacidad de institucionalizar en el Estado las demandas de la sociedad en torno a los problemas de integración generados y superables a través de la educación. Con ello, se avanza en la construcción de la infraestructura y orientaciones del grupo social expresado a través del movimiento.

Entonces ¿qué grupo social estaría siendo expresado a partir de este movimiento estudiantil? La hipótesis es que se trata del segmento sociológicamente etiquetado como nueva clase media. Esta clase o grupo social se distingue del concepto tradicional de clase puesto que su base estructural, si se quiere, no está en la producción directa, sino que en organizaciones educativas, prestadoras de servicio y que en general administran sus respectivos ámbitos de funcionamiento a través de medios burocráticos. Entonces el concepto de nueva clase media se diferencia de las tradicionales clases medias que en verdad estaban en una posición intermedia entre la clase obrera y la burguesía propietaria, es decir pequeños propietarios, pequeña burguesía, clase media independiente, etc. La nueva clase media corresponde a un grupo amplio y heterogéneo en cuanto a condiciones económicas e intereses, sobre el que incluso resulta forzado usar el concepto del clase, pero que comparte la condición de trabajadores asalariados, en general personal administrativo en una perspectiva amplia, en el sector de servicios. En otro sentido, la nueva clase media sería también nueva en Chile por la transformación y progresiva ampliación del grupo en los últimos treinta años, a través de todos los estratos socioeconómicos, en la medida que el sector de servicios se ha extendido para constituirse como el más amplio del empleo -67,2\% según CASEN del año 2000 (Arriagada, 2007)-. El sostenido proceso de tercerización de la economía nacional ha estado lógicamente asociado a la transformación a escala global de las relaciones de producción por medio de la introducción de las tecnologías de la información y del rol de la gestión del conocimiento en desarrollo organizacional. Ello también es un elemento de novedad para la clase media, ya que los profesionales (egresados de instituciones de educación superior) se constituyen en los trabajadores calificados del sector de servicios y su consecutiva expansión como segmento conduce, a su turno, a la masificación de los trabajadores intelectuales, quienes definen su quehacer en torno a la producción simbólica o de sentido, frecuentemente a través de medios administrativos. Este segmento profesional es el que determina hegemónicamente el contenido de las orientaciones (o aspiraciones) de la nueva clase media. 
Los intelectuales han sido tradicionalmente asociados a una elite, que ejerce el liderazgo moral en la sociedad. Gramsci amplio el concepto de intelectuales para abarcar en general a los "agentes de la superestructura” (Portelli, 1978), es decir todos los actores involucrados finalmente con la reproducción cultural y simbólica de la sociedad capitalista. Pero para Gramsci los intelectuales estaban en una relación orgánica con las clases sociales, en el sentido que finalmente pertenecían a la burguesía o al proletariado. Entonces a escala global el desarrollo del capitalismo de Estado, y el efecto transformador del Estado de Bienestar sobre la estructura social, condujo a la expansión del segmento de intelectuales por medio de la nueva clase media, al punto que el segmento en general se constituye como un grupo por sí mismo, con autonomía en relación a las otras clases sociales y que por lo tanto es capaz de articularse en torno a sus propios intereses.

En Chile a final de la década de los 60 e inicios de los 70 se constata una fuerte expansión de la matrícula universitaria en beneficio directo de los grupos medios, y tendiente a la formación de un fuerte estrato de intelectuales. El análisis de este proceso, por ejemplo de Garretón (1978) y Brünner (1979), deja planteada la tesis que estos grupos impulsaron y se apropiaron del proceso de reforma universitaria y en general de la expansión de la educación superior y formación profesional siguiendo sus propio intereses. Sin embargo, la dictadura representó una regresión de la solidaridad de la clase media y en la conformación del grupo de intelectuales, principalmente en la medida que las universidades fueron reducidas en su tamaño (Brünner, 1987), controladas, reprimidas, fragmentadas, mercantilizadas, al igual que sus disciplinas, principalmente aquellas críticas de las ciencias sociales que experimentaron mayor expansión durante el periodo anterior de reforma. Por otro lado, las políticas neoliberales implementadas en este período condujeron a la contracción del Estado, reduciendo con ello la participación de los sectores medios en la burocracia pública, y a la instalación del mercado -a través del trabajo asalariado e independiente (en menor medida) en el sector servicios y del consumocomo uno de los principales mecanismos de integración social para este grupo (Angelcos et. al., 2006). Por último y como aspecto decisivo, la educación, y especialmente la educación superior, pasa a ser provista a partir de este período principalmente a través del mercado, de modo que la formación, reproducción y ampliación de todo el grupo se lleva a cabo por medio de los mecanismos de consumo, que están diferenciadamente distribuidos de acuerdo a la estructura socioeconómica.

Pero de todas formas, a contar de la década de los noventa y con un sistema de educación superior mercantilizado, se observa, de acuerdo a los datos de la encuesta CASEN (Orellana, 2011), la sostenida expansión de la matrícula, cubriendo actualmente a aproximadamente el $45 \%$ de la cohorte que, excluyendo la deserción, corresponderán a los profesionales y técnicos del país, en una razón 7:3.

La masificación de la educación superior en Chile ha conducido a la constitución de un sector significativo de intelectuales en un sentido am- 
plio, que provienen de diferentes estratos socioeconómicos, y que probablemente su inserción laboral también redunda en segmentación socio económica, pero que comparten, en su condición de estudiantes y profesionales, el mismo principio de status social a través de credenciales y la ocupación en funciones equivalentes o intercambiables en el mundo del trabajo. Es decir, la identificación del movimiento estudiantil con el desarrollo de la nueva clase media y de trabajadores intelectuales en Chile se realiza desde el origen concreto de los integrantes del movimiento, es decir los estudiantes, en familias de clase trabajadora, incluyendo empleados, obreros y trabajadores no calificados, que efectivamente corresponden hoy al segmento mayoritario en educación superior, imprimiendo así carácter popular al segmento y determinando sus orientaciones. El carácter masivo del grupo se expresa también en la diferenciación de los tipos de formación, las orientaciones técnicas e ideológicas, más o menos asociados a la diversidad de los respectivos contextos socioeconómicos. Este es probablemente uno de los aspectos más notables de la actualidad y uno de los rasgos decisivos de la estructura social en Chile. Entonces, la perplejidad que ha producido la irrupción del movimiento, en circunstancias que nunca antes en el país se había abierto tal acceso a la educación, se sosiega en la constatación que justamente este acceso masivo es el que ha proporcionado la plataforma del movimiento y ha puesto en evidencia la comunidad de intereses, incluso en un sistema de educación escolar y superior segmentado y heterogéneo como es el chileno.

\section{Orientaciones y intereses}

La sociología contemporánea, a través de diversos autores (Mills, 1953; Dahrendorf, 1964; Touraine, 1974; Giddens, 1975; Gouldner, 1979; Parkin, 1981; Offe, 1987; Inglehart, 1990; Wright, 1999; Szelényi, 2004), ha observado la emergencia de la nueva clase media e intelectual en la sociedad occidental, aunque sin necesariamente ofrecer un relato unívoco acerca de sus orientaciones e intereses. Ello probablemente por el carácter heterogéneo y transversal del grupo en cuestión. Sin embargo se pueden destacar algunos elementos relevantes. En relación a la nueva clase media se le ha reconocido como portadora de valores post-materiales, como democracia, solidaridad, igualdad de género y autonomía, lo que tiende a asociarla a la izquierda de la política y a distinguirla de la clase media tradicional, anclada en valores materiales como la seguridad material y el logro económico y por ende asociada a la derecha política. Los valores post-materiales además estarían a la base de los nuevos movimientos sociales. Este mismo factor puede ser haber sido determinante en la dificultad que ha demostrado la nueva clase media para organizarse políticamente en la forma de partidos e incluso en sindicatos (Fleet, 2011). En la perspectiva de la llamada nueva clase intelectual se ha planteado que son las portadoras de una cultura discursiva (como opuesta a la coerción y potencialmente emancipatoria), y que principalmente tienen en el Estado, y sus medios administrativos, la plataforma para acceder a posiciones dominantes y ofrecer una alternativa a la sociedad 
subordinada al capital. En efecto, espacios que el Estado ha protegido del capital, como la universidad, han catalizado los conflictos sobre el uso del conocimiento y la dirección de la sociedad. En esta misma línea, teóricos de tradición crítica, como Habermas (1980), han visto a las comunidades universitarias, en especial a los estudiantes, como uno de los pocos ámbitos de la sociedad donde aún se conserva el potencial de protesta y resistencia ante de fuerza integradora y manipuladora de la sociedad capitalista avanzada.

Desde luego que estas clases medias e intelectuales, tal y como han sido descritas y analizadas para el contexto fundamentalmente europeo, no necesariamente se han desarrollado de la misma manera en Chile. No pretendo aquí establecer un análisis comparado de ambas situaciones, pero si afirmar que sectores medios e intelectuales han tenido, desde la constitución de distintas versiones del Estado de bienestar o compromiso, un desarrollo sostenido por referencia al Estado, mientras que en el caso chileno el período de la dictadura y las reformas neoliberales introducidas en el país con ella, constituyen una clara discontinuidad que ha distanciado a estos grupos del Estado como empleador y garante de la educación pública como ámbito privilegiado de formación. En este sentido la demanda central de movimiento estudiantil hacia la recuperación del carácter público de la educación, reivindicando el papel del Estado en restituir su valor de uso y proscribir su mercantilización (fin de lucro), representa el potencial reencuentro histórico de la clase media e intelectual con sus intereses propiamente generalizables.

La hipótesis de este punto es que la articulación entre demandas estudiantiles y las orientaciones colectivas de su base social se produce en la pretensión de ejercer control reflexivo sobre las condiciones de formación y reproducción de la clase. De manera sintética, la falta de control sobre el sistema de educación se expresa en condiciones segmentación, subordinación y problemas de calidad para la reproducción de los sectores medios.

Primero entonces, el sistema de educación Chile en su conjunto actualmente está determinado por el valor de cambio, es decir, diferencia tipos de proveedores y servicios educativos en términos de precio, generando de esta manera un mercado que, como cualquier otro, segmenta niveles de calidad, y que constituye al mismo tiempo mercados específicos para sus servicios y recursos relacionados, como la docencia y, particularmente en la educación superior: investigación, académicos, servicios, estudiantes, gestión organizacional, créditos, publicidad, etc. (Brünner y Uribe, 2007).

Segundo, la mercantilización sitúa al lucro en la centralidad del conflicto, pues expresa, de manera más clara en la educación superior, quienes sí son los que controlan el sistema o mercado de la educación, porque se apropian del excedente generado por este mercado y sus mercados asociados, y se ponen así en una posición dominante en relación la clase social 
para la que la educación es un requisito para constituirse como tal. Además, la educación como medio de acumulación privada para los proveedores, aunque sea de manera latente en el caso de la educación superior, termina introduciendo al interior del Estado subsidiario la contradicción entre intereses que pueden finalmente ser incompatibles: por un lado, la educación como interés general y que por lo tanto se define por su valor de uso, es decir, la calidad y, por el otro, la educación como negocio, es decir que hace posible el servicio siempre que el valor de cambio posibilite margen de ganancia. Tal contradicción es primero visible como un problema de racionalidad de las políticas públicas subsidiarias, que entregan recursos limitados a la educación pero al mismo tiempo hacen posible la formación de mercados que finalmente dan prioridad a los intereses particulares constituidos en torno al lucro (por ejemplo, el notorio caso del Crédito con Aval del Estado), los cuales al apropiarse de parte de estos recursos, merman el potencial de efectividad de tales políticas. Como resultado entonces se constituye el problema político para el Estado, tal como ha estado ocurriendo, porque no es capaz de administrar el interés público sin subordinarlo a los intereses privados, y así el ámbito mismo de formulación de las políticas públicas se convierte en espacio de enfrentamiento de posiciones y movilización política, terminando en todo lo contrario al carácter técnico y no político que las orientaciones neoliberales pretendieron instaurar en el Estado chileno.

Por último, al comportarse la educación y servicios asociados como mercados autorreferenciados, la provisión de la oferta se lleva a cabo sin que medie necesariamente la autorregulación o la consideración de las necesidades colectivas (tal como Weber caracteriza el capitalismo, mercados cuya racionalidad es formal u operativa y que se impone por sobre la racionalidad material en tanto orientación a satisfacer los requerimientos de la sociedad). Los efectos de esta situación son conocidos de tanto en tanto como problemas de la calidad. A continuación solo cuatro ejemplos. La falta de pertinencia y sobreoferta de distintos programas de pregrado: según datos del Consejo Nacional de Educación la carrera de educación media es la que tiene mayor matrícula en todo el sistema de educación superior en Chile, con el 5\% del total; las carreras de pedagogía constituyen el $11,8 \%$ del total de oferta de carreras de pregrado, abarcado el 17\% de todos los estudiantes universitarios del país, y siendo el segmento de carreras con mayor presencia (95\%) de egresados de educación escolar municipal y particular subvencionada. La baja calidad de sedes: en 2009 habían 141 sedes de instituciones de educación superior no declaradas, correspondientes principalmente a universidades privadas (CNA, 2010). Poca efectividad del sistema en cuanto a permanencia: la duración real de las carreras de pregrado en Chile promedia los 11,3 semestres (Divesup, 2010); y alta deserción: la deserción hacia el tercer año es de 40\% en universidades, 60\% en los institutos profesionales y 52\% en centros de formación técnica (CSE, 2008). Finalmente, precariedad en el desarrollo de los cuerpos académicos: el 50\% del total de profesores universitarios son contratados a honorarios por horas de clase, mientras que solo el $21 \%$ tienen contrato por jornada completa. 
Estas condiciones de mercado bajo las que se ha reproducido la nueva clase media en los últimos treinta años en Chile no solo han sido determinantes para la formación de sus bases estructurales, sino que también de sus referentes simbólicos o, de manera más general, de sus valores. La construcción social del grupo ha operado privadamente, en nombre de intereses particulares que ejercen elecciones formalmente libres, pero estructuralmente determinadas por las diferencias sociales pre-existentes, en términos de capacidad adquisitiva, condiciones de vida, valores, etc. (Ball et. al., 2002; González, 2011). En el caso de la educación escolar, la opción de educación pública y gratuita corresponde a una alternativa residual, en el sentido de la política asistencialista propiamente neoliberal de ofrecer servicios donde el mercado no dispone de proveedores. En el caso de la educación superior, el sector público provee de servicios con arreglo a la misma lógica de mercado que rige en el sector privado. En estas circunstancias, la clase se reproduce de manera espontánea, transmitiendo los respectivos patrimonios económicos y culturales de cada sub-grupo a las futuras generaciones, y el sistema de educación en el fondo cumple la función de transformar el privilegio en mérito. Aún más, y creo que en este punto hay elemento peculiar del sistema chileno, muchas de las distintas instituciones de educación superior no solo acceden a segmentos de estudiantes, que ya están previamente diferenciados por la educación escolar que han recibido, sino que también imprimen valores e ideologías específicas. La educación superior chilena tiene instituciones públicas, privadas y confesionales, para distintos estratos socioeconómicos, agregando así mayor diferenciación interna al grupo social, y particularmente fragmentación de conciencia, en el sentido que los profesionales son formados en diferentes ideologías y credos religiosos que con alta probabilidad se corresponden con el poder que terminan ejerciendo dentro de las organizaciones, las funciones que desempeñan, los salarios que obtienen y sus respectivas pertenencias a grupos socioeconómicos. En otras palabras, las universidades e instituciones de educación superior se especializan en la formación de la vocación de la élite dirigente católica, la élite Opus Dei y la élite emprendedora; de la burocracia con sentido público, la burocracia con sentido empresarial y la burocracia con orientación religiosa al más pobre; los profesores para los colegios particulares, los colegios subvencionados y los colegios municipales; los ingenieros y administradores para las grandes empresas, y aquellos para las pequeñas; médicos para las clínicas y médicos para hospitales y consultorios; etc.

La voluntad de reconstrucción de un sistema de educación público, a cargo del Estado, está animada - o alineada - por el interés de los grupos medios e intelectuales de institucionalizar el principio universalista, o democrático si se quiere, en su formación como clase social. Ello es incompatible con la segmentación de mercado, “a espaldas” del grupo, y desde luego con el lucro como subordinación de la producción de este grupo a otros intereses dominantes. Finalmente, el control que los sectores intelectuales y medios puedan ejercer sobre su propia reproducción a través de la educación pública va en la dirección de adquirir consciencia y potencialmente emancipar a los sujetos del efecto limitante de los condicionantes subjeti- 
vos de la acción, como el "capital cultural” (Bourdieu, 1986) o "códigos restringidos” (Bernstein, 1970).

Orientaciones de clases medias e intelectuales, como las hipotetizadas por la sociología, se perfilan como potencial proyecto adelantado a través de las movilizaciones estudiantiles. El interés particular de clase de constituir una base social consistente y legítima del grupo a través del control reflexivo sobre la educación pública es generalizable como propuesta de integración para el conjunto de la sociedad. A esto se agrega la protección de la educación pública, especialmente de la educación superior, como esfera de la razón que produce los discursos (científico, normativo, estético) sobre la sociedad libre de la coacción del poder y del dinero. La decomodificación de la educación es entonces una condición para desplegar su potencial crítico. Por otra parte, el conjunto de medios administrativos que aplican sobre la educación, en particular mecanismos de regulación, pueden beneficiarse de contenido sustantivo y superar formulaciones puramente tecnocráticas, como por ejemplo la indefiniciones que actualmente predominan en los discursos sociales y políticos sobre calidad de la educación y requerimientos de profesionales. Por último, el proyecto de los sectores medios e intelectuales se expresa a través de las movilizaciones estudiantiles en la medida en que el aporte basal demandado para las universidades se puede traducir en el fortalecimiento de la infraestructura de investigación, considerando la formación de investigadores y el incremento en el número de proyectos, favoreciendo la competitividad del país en cuanto a su participación en la economía globalizada del conocimiento.

La disposición a instrumentalizar los medios administrativos del Estado para llevar adelante las demandas del movimiento estudiantil hacia la educación pública, y con ello acceder al control de las bases de reproducción social, puede también conducir a restituir la orientación hacia el interés general, de manera directa o sustantiva, en el Estado mismo.

\section{Intelectuales, nueva clase media y capitalismo: ¿Proyecto alternativo?}

La crisis del capitalismo liberal hacia 1930 en el mundo, y también en Chile, condujo a la redefinición de las bases políticas y sociales para el desarrollo del capitalismo, lo que fue institucionalizado como el compromiso de clases, entre capital y trabajo, en el Estado. Tal compromiso fue la salida a la crisis del sistema económico que se trasladó a una crisis social, lo que a su turno es, como expuse en el primer punto, una crisis de legitimidad. Como resultado de este nuevo orden, y del rol del Estado en la decomodificación de las condiciones de reproducción de la clase trabajadora, se produjo la expansión de la nueva clase media e intelectuales, cuya identidad estuvo definida por derecho universales implementados a través del Estado, que eran la proyección de sus intereses generalizables. En Chile, el quiebre del modelo del Estado de compromiso, producido principal- 
mente por la dificultad de garantizar su propia legitimidad por medio de la combinación de la función de integración social con la promoción de la acumulación capitalista de acuerdo a intereses privados, se completó a través de la privatización, es decir re-comodificación, de las bases de reproducción de los sectores medios y de la sociedad en general. Pero en este orden, adoptado en Chile hace ya más de treinta años, los sectores medios e intelectuales se han seguido expandiendo, y la educación privatizada y mercantilizada ha sido uno de los principales medios para ello. El mercado justamente ha conducido a que el trabajo intelectual no sea solamente un rasgo distintivo de las elites (como grupo social que ejerce el poder en nombre de la clase dominante) sino que constituye hoy un ámbito de formación y desempeño masivo, atravesando los diferentes estratos.

Sin embargo, hoy es claro que la expansión de la educación superior sostenida fundamentalmente en el gasto privado tocó techo (OCDE, 2009). Al mismo tiempo, el estadio de masificación alcanzado por la educación superior en Chile la ha convertido no solo una condición de membresía de clase, sino que en un requisito fundamental de entrada al mundo del trabajo (Trow, 2007). El rol que la educación superior tiene hoy en determinación de las condiciones de ingreso al mercado laboral es similar al que hace treinta años tenía la educación media. Es este escenario el que convoca, desde el punto de vista de los intereses materiales, a la emergencia del movimiento estudiantil.

Para considerar al movimiento estudiantil como adelanto del proyecto de la nueva clase media y de los intelectuales no es necesario que exista actualmente una articulación consciente e institucionalizada, en la forma de pacto político, sino que sencillamente ello resultaría del eventual éxito en la recuperación de la educación pública. Ésta sería la principal plataforma de poder del grupo social, la base para su construcción material y simbólica a pesar de las actuales segmentaciones internas, y para participar en la definición de los fines de la sociedad en su conjunto. Aunque de todas maneras, el posible desarrollo político de un proyecto alternativo de clase requeriría niveles de organización y articulación también políticos, de manera de ejercer el poder de manera sistemática sobre las instituciones que lo distribuyen, principalmente el Estado. Sin embargo, como vimos en el primer punto, el momento actual muestra el divorcio entre estas orientaciones y potencial proyecto, por un lado, y los partidos y grupos políticos, por el otro. Llama en verdad la atención la carencia de actores políticos directamente orientados a movilizar los grupos medios e intelectuales durante los últimos años, pero independientemente de todos los factores que puedan explicar este fenómeno, notablemente dentro de ellos la Constitución de 1980, la reproducción del poder por parte de los políticos (y su presencia como administradores del sistema de decisiones) ha dependido menos de la interacción con la sociedad y más de las interacciones repetidas entre los mismos políticos en el poder, aunque en la opinión de algunos analistas (Aninat et. al., 2006) ello justamente ha sido uno de los factores decisivos en la eficiencia del proceso de toma de decisiones en Chile. 
Categorías sociales como profesionales, jóvenes, estudiantes, empleados, funcionarios, profesores, que muestran la diferenciación de la sociedad chilena en las últimas décadas, tienen en la educación pública la plataforma para articular el conflicto principal de la sociedad chilena, por la defensa de su espacio de reproducción material y simbólica (su mundo de la vida) del mercado, y la oposición a los grupos dominantes que en último término dominan a través de él. El proyecto alternativo emergería cuando este conflicto se traduzca en el cuestionamiento sobre la institucionalización de las relaciones de dominación en la sociedad, como la demanda por el cambio de Constitución, y en la lucha por determinar sus fines. Las distintas expresiones de protesta que no solo en Chile sino que a escala global se dirigen contra el modelo de capitalismo financiero, y las políticas neoliberales que lo sustentan, sugieren que sus dificultades de orden sistémico están haciendo resonancia a nivel social -movimiento social o ciudadanos mediante- de tal manera que una posible crisis de legitimidad es anunciada. En tales circunstancias, es esperable que la redefinición del pacto que en el futuro determine las bases de la acumulación económica y distribución del poder en la sociedad incorporen esta vez - a diferencia del compromiso del Estado de Bienestar, y del "consenso" neoliberal - a la nueva clase media y trabajadores intelectuales. A su turno, la capacidad de estos grupos para ejercer sus prerrogativas en el contexto de un nuevo orden vincula el nivel de movilización, política y social, con las posiciones que hayan ido consolidando en la sociedad. En otras palabras, la capacidad de movilización y de acción colectiva se construye en torno a la independencia consolidada en relación al mercado (Esping-Andersen, 1988). Por esta razón los estudiantes tienen mayor capacidad de movilizarse que los trabajadores en Chile, y entonces pueden actuar como agentes de las demandas de estos últimos. La educación pública es sin duda una posición fundamental en el sentido de construir la independencia de la sociedad con respecto al mercado, pero no la única, si se tiene en cuenta, además de la "agenda" redistributiva que considera reforma tributaria y renacionalización del cobre, el potencial control que pueden ejercer sobre la economía nacional si es que la lucha se dirigiera a reivindicar el poder de decidir dónde invertir los fondos de pensiones, hoy acumulados y administrados por empresas AFP a espaldas de los verdaderos dueños de su capital.

Para concluir, un proyecto alternativo finalmente se constituye como tal si propone un nuevo modelo de desarrollo para la sociedad, el cual ponga la actividad que define al grupo emergente, en este caso el trabajo asalariado e intelectual en un sentido amplio, en el centro de la producción y la distribución, y con la respectiva capacidad de reclamar reconocimiento y participación en el poder. En este sentido, la emergencia de un proyecto alternativo que a la vez proponga un modelo de desarrollo es el potencial efecto de un conflicto social central (o societal) sobre el valor del trabajo y el uso del conocimiento, y contra la desigualdad entendida como dominación y explotación (no solo como un problema de los estándares del país en relación al "efecto demostración" de la OCDE, desde un punto de vista tecnocrático). Aunque no se trata de especular la forma que podría adquirir la reorganización de las actividades económicas, si se puede suponer que 
un modelo de desarrollo en tal sentido implicaría resituar el trabajo asalariado, de manera general, como fuente de valor, y para ello el Estado nuevamente tendría que asumir su protección (a través de derechos) y fortalecimiento a nivel interno (a través de políticas redistributivas).

La educación está siendo el espacio preciso para criticar la hegemonía y el modelo de desarrollo mercantil imperante, a la vez que para plantear su superación. Lo que ha sido llamado la economía del conocimiento a escala global justamente refiere a la importancia relativa adquirida por el ámbito de la producción cuya rentabilidad económica depende de la gestión del conocimiento; pero ello no ha conducido a que la educación y sus instituciones sean las que definan la dirección y contenido de este modelo económico, sino que es "la subordinación de la universidad al capital, y al Estado en nombre del capital” (Marginson, 2006: 46). En consecuencia, la recuperación de la educación pública para el país, como es demandado por el movimiento estudiantil, también apunta a la reapropiación social del potencial de desarrollo económico abierto por la educación y sus instituciones productoras de conocimiento, y la liberación de este ámbito del efecto regresivo del capital. 


\section{Nota}

${ }^{1}$ El autor agradece los comentarios y observaciones de Dr. David Lehmann, Jorge Vásquez y Javiera Muñoz, que han contribuido a enriquecer los argumentos aquí planteados. 


\section{Bibliografía}

Angelcos, N., Pérez, P., y Sémbler, C., (2006), “Los sectores medios en la era neoliberal: transformaciones y contradicciones del desarrollo en Chile”, en Revista de Sociología de la Universidad de Chile, $n^{\circ} 20$, Universidad de Chile, Santiago de Chile.

Aninat, C., Langredan, J., Navia, P. y Vial, J. (2006), Political institutions, policymaking processes and policy outcomes in Chile, BID, Washington D.C.

Arriagada, I. (2007), “Abriendo la caja negra del sector servicios en Chile y Uruguay”, en Gutierrez, M. A. Género, familias y trabajo: rupturas y continuidades, CLACSO, Buenos Aires.

Ball, S., Davies, J., David, M. y Reay, D., (2002), “Classification and judgement: social class and the cognitive structures of choice of higher education”, en British Journal of Sociology of Education, vol. 23, $n^{\circ} 1$, 5172, Taylor \& Francis, Londres.

Bernstein, B. (1970), “Education cannot compensate for society”, en School and society: a sociological reader, Roultledge \& Kegan Paul and The Open University Press, Londres.

Bourdieu, P. (1986), “The forms of capital”, en Richardson, J., Handbook of Theory and Research for the Sociology of Education, Greenwood, Westport.

Brünner, J. J., (1979), Universidad, cultura y clases sociales, FLACSO, Santiago.

Ídem (1987), Educación superior en Chile: entre el Estado, el mercado y los intereses académicos. FLACSO, Santiago.

Brünner, J. J., y Uribe, D., (2007), Mercados universitarios: el nuevo escenario de la educación superior, Universidad Diego Portales, Santiago.

CNA (2010), Sedes de instituciones de educación superior en Chile, working papper Santiago.

CSE (2008), Retención en el sistema de educación superior: seguimiento de las cohortes 2004, 2005 y 2006, working paper, Santiago.

Dahrendorf, R. (1964), "Recent changes in the class structure of European societies” en Daedalus, Vol. 93, n 1 , MIT Press, Massachusetts.

Esping-Andersen, G. (1988), Politics against markets. The social democratic road to power, Princeton University Press, New Jersey.

Fleet, N. (2009), “Razón y dominación. La teoría de la legitimidad de Max 
Weber como orientación simbólica de la acción política” en Revista Austral de Ciencias Sociales, $n^{\circ} 16$, Universidad Austral de Chile, Valdivia.

Ídem. (2011), "Does the growth of a post-materialist middle class help labour-based parties to overcome the 'dilemma of electoral socialism'?” en Composicao, Revista de Ciencias Sociais, $n^{\circ}$ 8, Universidad Federal de Mato Grosso do Sul, Mato Grosso do Sul.

Garretón, M. A. (1978), Universidad y política en los procesos de transformación y reversión en Chile 1967-1977, FLACSO, Santiago.

Giddens, A. (1975), The class structure of the advanced societies, Harper \& Row, Londres.

González, J. (2011), Baja participación en educación superior: hallazgos en la toma de decisiones de los jóvenes de menor nivel socioeconómico, Centro de Estudios de Políticas y Prácticas en Educación, Pontificia Universidad Católica de Chile, Santiago de Chile.

Gouldner, A. (1979), The future of intellectuals and the rise of the new class, The Macmillan Press London.

Habermas, J. (1980), Toward a rational society, Heinemann Educational Books, Londres.

Inglehart, R. (1990): Culture Shift in advanced industrial society, Princeton University Press, Princeton.

King, L. P. y Szelényi, I. (2004), Theories of the new class: Intellectuals and power, University of Minnesota Press, Minneapolis.

Lechner, N. (1983), Estado y política en América Latina, Siglo XXI, México.

Marginson, S. (2006), "Putting 'public' back into the public university", en Thesis Eleven, $n^{\circ} 84$, SAGE, Londres.

Mills, C. W. (1953), White collar, Oxford University Press, New York.

Ministerio de Educación (2010), Duración real de las carreras y/o programas. Descripción y análisis de la cohorte de titulados y/o graduados 2007, Ministerio de Educación, Santiago.

OCDE (2009), La educación superior en Chile: Revisión de Políticas Nacionales de Educación. OECD, Santiago de Chile.

Offe, C. (1987), “Challenging the Boundaries of Institutional Politics: Social Movements since the 1960s” en Maier, Ch. Challenging the boundaries of the political, Cambridge University Press, Cambdrige. 
Orellana, V. (2011), Caracterización social de los estudiantes de educación superior en Chile, Centro de Investigación en Estructura Social, Universidad de Chile, Santiago de Chile.

Parkin, F. (1981), Marxism and class theory. A bourgeois critique. Tavistock Publications, London.

Portelli, H. (1978): Gramsci y el bloque histórico, Siglo XXI, México D.F.

Touraine, A. (1974): The post-industrial society. Tomorrow's social history: classes, conflicts and culture in the programmed society, Random House, London.

Trow, M. (2007), "Reflections on the transition from elite to mass to universal access: forms and phases of higher education in modern societies since WWII" en Forest, J. y Altbach, P. G. (eds.) International Handbook of Higher Education, Springer, Dordrecht.

Wright, E. O. (1999), “What is middle about the middle class?” en Roemer, J. Analytical Marxism, Cambridge University Press, Cambridge. 